\title{
Wall Mitigation Techniques for Indoor Sensing within the Compressive Sensing Framework
}

\author{
Eva Lagunas ${ }^{\#}$, Moeness G. Amin ${ }^{*}$, Fauzia Ahmad ${ }^{*}$, Montse Nájar \\ \# Universitat Politècnica de Catalunya, Barcelona, Spain \\ \{eva.lagunas, montse.najar\}@upc.edu \\ ${ }^{*}$ Radar Imaging Lab, Center for Advanced Communications, Villanova University, Villanova, PA 19085, USA \\ 2 moeness.amin, fauzia.ahmad\}@villanova.edu
}

\begin{abstract}
Compressive sensing (CS) for urban operations and through-the-wall radar imaging has been shown to be successful in fast data acquisition and moving target localizations. However, the research work in this area thus far has assumed prior effective wall removal, allowing proper detection of indoor targets. In this paper, we show that wall removal techniques, operating with full data volume and applying backprojection imaging methods, can be equally effective under reduced data volume and within the sparse signal reconstruction framework. Specifically, we demonstrate that the spatial filtering and the singular value decomposition based approaches, which, respectively, exploit the spatial invariance and the strength of the EM wall return, for suppression of the wall reflections, can be employed using few measurements, thus allowing CS to be applied to data with higher target-to-wallclutter ratio.
\end{abstract}

\section{INTRODUCTION}

Through-the-Wall Radar Imaging (TWRI) is an emerging technology of rising interest with the objectives of sensing through the wall and inside enclosed building structures using EM waves. The sensing could determine the building layouts, discern the intent of activities inside the building, and detect, identify and track moving targets. This type of technology is highly desirable in search-and-rescue missions, behind-wall target detection, and surveillance and reconnaissance in urban environments [1]-[3]. In order to achieve these objectives, however, much attention should be paid to the front wall and its effects on the imaged scene accuracy and fidelity.

Front walls cause strong EM reflections back in the directions of the radar which obscure the behind-the-wall target returns. The latter are relatively weak compared to wall reflections and cannot be generally detected without an effective removal of the wall clutter. For moving targets, subtraction of data acquired at different times alleviates this problem and leads to removal of cross talk and wall EM reflections as well as suppression of stationary clutter inside the room [4]. However, when the targets of interest are themselves stationary, front wall reflections should be properly attenuated without the requirements of having a reference or background scene.

In this paper, we address wall mitigations in the context of Compressive Sensing (CS). For backprojection-based imaging and when all data measurements are available, three main approaches have been proposed to deal with strong wall EM reflections without relying on the background scene data [5]-
[8]. In the first approach, the parameters of the front wall, such as thickness and dielectric constant, are estimated from the first wave arrivals [8]. The estimated parameters can be used to model EM wall returns, which are subsequently subtracted from the total radar returns, rendering wall-free signals. The second approach applies a spatial filtering method for wall clutter mitigation [5]. The spatial filter removes the zero frequency, and relies on the wall returns being invariant with changing antenna location. The third approach recognizes the wall reflections as the strongest component of radar returns. By applying singular value decomposition (SVD) to the measured data matrix, the wall returns are captured by the singular vectors associated with the dominant singular values [6], [7] and removed by orthogonal subspace projection.

In this paper, we examine the performance of the wall mitigation techniques for sparse image reconstruction. Only a small subset of measurements is employed for image formulation. Data reductions in space or/and time allow fast data acquisition which, in turn, enables achieving situational awareness in a quick and reliable manner. The application of CS for TWRI was first reported in [9] and further developed in [10], [11]. However, wall mitigation in conjunction with CS has never been considered in these references.

We focus on the SVD-based and spatial filtering-based wall mitigation methods. It is shown that these methods maintain their proper performance with few measurements as their fulldata volume counterparts. This allows CS techniques to proceed and act on data with much reduced wall clutter.

The reminder of this paper is organized as follows: In Section II, the system model and conventional wall clutter mitigation methods are introduced. In Section III, the CS theory is applied to TWRI. Finally, Section IV presents experimental results, and Section $\mathrm{V}$ states the conclusions.

\section{Wall Clutter Mitigation}

\section{A. Signal Model}

Assume a synthetic aperture radar (SAR) is used in which a single antenna at one location transmits and receives the radar signal, then moves to the next location, and repeats the same operation along the axis parallel to a homogenous wall. Assume $N$ antenna locations and a stepped-frequency signal of $M$ frequencies, which are equispaced over the desired bandwidth $\omega_{M-1}-\omega_{0}$, 


$$
\omega_{m}=\omega_{0}+m \Delta \omega, m=0,1, \ldots, M-1
$$

where $\omega_{0}$ is the lowest frequency in the desired frequency band and $\Delta \omega$ is the frequency step size. With the wall reflection, the received signal at the $n$th antenna corresponding to the $m$ th frequency due to a single point target of complex reflectivity $\sigma_{p}$ is given by

$$
y(m, n)=\sigma_{w} \exp \left(-j \omega_{m} \tau_{w}\right)+\sigma_{p} \exp \left(-j \omega_{m} \tau_{p, n}\right)
$$

where $\sigma_{w}$ is the wall reflectivity, $\tau_{w}$ is the two-way traveling time of the signal from the $n$th antenna to the wall, and $\tau_{p, n}$ is the two-way traveling time between the $n$th antenna and the target. For through-the-wall propagation, $\tau_{p, n}$ will comprise the components corresponding to traveling distances before, through, and after the wall [12].

\section{B. Spatial Filtering}

From (2), we note that $\tau_{w}$ does not vary with the sensor location since the array is parallel to the wall. This implies that the first term in (2) assumes the same value across the array aperture. Unlike $\tau_{w}$, the time delay $\tau_{p, n}$ in (2) is different for each antenna location, since the signal path from the antenna to the target is different from one antenna to the other. For the $m$ th frequency, the received signal is a function of $n$ via the variable $\tau_{p, n}$. Therefore, we can rewrite (2) as,

$$
y_{\omega_{m}}(n)=v_{\omega_{m}}+u_{\omega_{m}}(n)
$$

where $v_{\omega_{m}}=\sigma_{w} e^{-j \omega_{m} \tau_{w}}$ and $u_{\omega_{m}}(n)=\sigma_{p} e^{-j \omega_{m} \tau_{p, n}}$. Thus, separating wall reflections from target reflections amounts to basically separating constant (zero-frequency signal) from non-constant valued signals across antennas, which can be performed by applying a proper spatial filter [5].

In its simplest form, the spatial filter, which notches out the zero spatial frequency component, can be implemented as the subtraction of the average of the radar return across the antennas. That is,

$$
\tilde{y}_{\omega_{m}}(n)=y_{\omega_{m}}(n)-\bar{y}_{\omega_{m}}, \quad \bar{y}_{\omega_{m}}=\frac{1}{N} \sum_{n=0}^{N-1} y_{\omega_{m}}(n)
$$

The filtered data will have little or no contribution from the wall reflections.

\section{SVD Approach}

The signals received by the $N$ antennas at the $M$ frequencies are arranged into an $M \times N$ matrix, $\mathbf{B}$,

$$
\mathbf{B}=\left[\begin{array}{lllll}
\mathbf{b}_{0} & \ldots & \mathbf{b}_{n} & \ldots & \mathbf{b}_{N-1}
\end{array}\right],
$$

where $\mathbf{b}_{n}$ is the $M \times 1$ column vector containing the steppedfrequency signal received by the $n$th antenna,

$$
\mathbf{b}_{n}=\left[\begin{array}{lllll}
y(0, n) & \ldots & y(m, n) & \ldots & y(M-1, n)
\end{array}\right]^{T} .
$$

The eigen-structure of the imaged scene is obtained by performing the SVD of B,

$$
\mathbf{B}=\mathbf{U D V} \mathbf{V}^{H},
$$

where $H$ denotes the Hermitian transpose, $\mathbf{U}$ and $\mathbf{V}$ are unitary matrices containing the left and right singular vectors, respectively, and $\mathbf{D}$ is a diagonal matrix containing the singular values $\lambda_{1}, \lambda_{2}, \ldots, \lambda_{N}$ in decreasing order, i.e., $\lambda_{1} \geq \lambda_{2} \geq \ldots \geq \lambda_{N}$.

The SVD based method assumes that the wall returns and target reflections lie in different subspaces. Therefore, the first $K$ dominant singular vectors of the $\mathbf{B}$ matrix are used to construct the wall subspace,

$$
\mathbf{S}_{\text {wall }}=\sum_{i=1}^{K} \mathbf{u}_{i} \mathbf{v}_{i}^{H} .
$$

The subspace orthogonal to the wall subspace is,

$$
\mathbf{S}_{\text {wall }}^{\perp}=\mathbf{I}-\mathbf{S}_{\text {wall }} \mathbf{S}_{\text {wall }}^{H},
$$

where $\mathbf{I}$ is the identity matrix. To mitigate the wall returns, the $\mathrm{B}$-scan matrix is projected on the orthogonal subspace

$$
\widetilde{\mathbf{B}}=\mathbf{S}_{\text {wall }}^{\perp} \mathbf{B} \text {. }
$$

In Section IV, we show that both spatial filtering and subspace methods for wall mitigation can proceed with reduced measurements and can thus serve as a preprocessing step to CS algorithms. In essence, the operations underlying both methods are not compromised by using fewer frequencies and antennas.

\section{COMPRESSIVE SENSING FOR TWRI}

Assume that the region of interest is divided into a finite number of pixels, $N_{x} \times N_{z}$, in crossrange and downrange, and that the targets occupy no more than $P\left(<<N_{x} \times N_{z}\right)$ pixels. Let $s(k, l), k=0,1, \ldots, N_{x}-1, l=0,1, \ldots, N_{z}-1$, be a weighted indicator function, which takes the value $\sigma_{p}$ if the $p$ th point target exists at the $(k, l)$-th pixel; otherwise, it is zero. If the values $s(k, l)$ are lexicographically ordered into a column vector $\mathbf{s}$ of length $N_{x} N_{z}$, the preprocessed received signal corresponding to the $n$th antenna and the $m$ th frequency can be expressed as,

$$
\tilde{y}(m, n)=\boldsymbol{\psi}_{m n} \mathbf{s},
$$

where $\boldsymbol{\psi}_{m n}$ is a row vector of length $N_{x} N_{z}$, and is given by

$$
\boldsymbol{\psi}_{m n}=\left[\exp \left(-j \omega_{m} \tau_{n, 00}\right), \ldots, \exp \left(-j \omega_{m} \tau_{n, N_{x} N_{z}}\right)\right] .
$$

Similarly, the vector $\tilde{\mathbf{y}}$, obtained by a lexicographical ordering of all preprocessed measurements, can be expressed as,

$$
\widetilde{\mathbf{y}}=\boldsymbol{\Psi} \mathbf{s}
$$

where $\boldsymbol{\Psi}$ is an $M N \times N_{x} N_{z}$ matrix whose rows are the vectors $\boldsymbol{\psi}_{m n}$ defined in (12). The expression in (13) considers the full dataset, consisting of the measurements made at the $N$ array locations using the $M$ frequencies. As the scene is sparse, it is possible to recover $\mathbf{s}$ from a reduced set of measurements. Consider $\xi$, which is a vector of length $Q_{m} Q_{n}(<M N)$ consisting of elements randomly chosen from $\widetilde{\mathbf{y}}$ as follows,

$$
\xi=\Phi \widetilde{y}=\Phi \Psi \mathbf{s}
$$



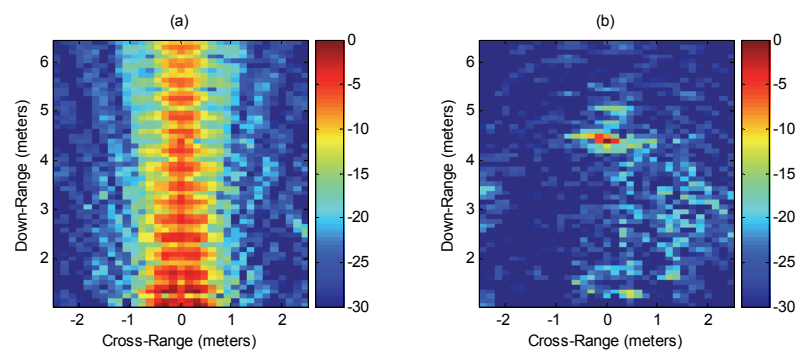

Fig. 1. TWRI images of the far target scene: (a) no preprocessing, (b) after background subtraction.
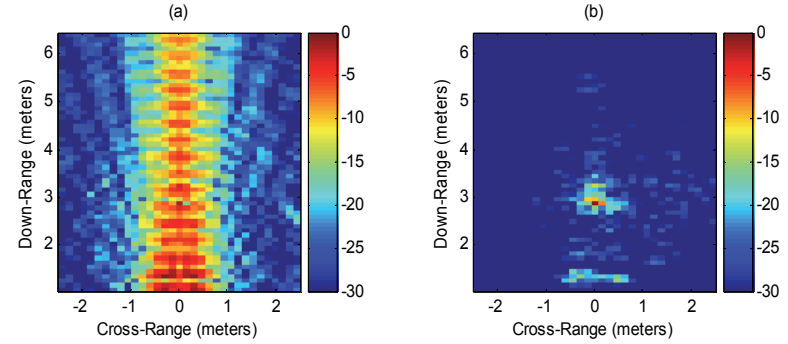

Fig. 2. TWRI images of the close target scene: (a) no preprocessing, (b) after background subtraction.

where $\boldsymbol{\Phi}$ is a matrix of size $Q_{m} Q_{n}$ x $M N$ that contains no more than one non-zero element in each row and each column. Given $\xi$, we can recover $\mathbf{s}$ by solving the following equation,

$$
\hat{\mathbf{s}}=\arg \min _{x}\|\mathbf{x}\|_{l_{1}} \text { subject to } \quad \xi \approx \mathbf{\Phi} \Psi \mathbf{x}
$$

The selected frequency bins should be distributed over the entire frequency band and the selected sensors should cover the extent of the array.

\section{EXPERIMENTAL RESULTS}

A through-the-wall SAR system was set up in the Radar Imaging Lab at Villanova University. A line array of length $1.2446 \mathrm{~m}$ with $0.0187 \mathrm{~m}$ inter-element spacing was synthesized, parallel to a $0.14 \mathrm{~m}$ thick solid concrete wall, at a standoff distance of $1.01 \mathrm{~m}$. The back and the side walls of the room were covered with RF absorbing material to reduce clutter. A stepped-frequency signal covering 1 to $3 \mathrm{GHz}$ band with 2.75 $\mathrm{MHz}$ frequency step was employed. A vertical dihedral was used as the target and was placed once at $3.04 \mathrm{~m}$ and then at $1.52 \mathrm{~m}$ on other side of the front wall. The size of each face of the dihedral is $0.39 \mathrm{~m}$ by $0.28 \mathrm{~m}$. The empty scene without the dihedral target present was also measured for comparison.

The region to be imaged is chosen to be $4.9 \mathrm{~m} \times 5.3 \mathrm{~m}$ centered at $(0,3.65) \mathrm{m}$ and divided into $33 \times 57$ pixels in crossrange and down-range. Fig. 1(a) and Fig. 2(a) show the images corresponding to both scenes obtained with conventional backprojection applied directly to the received signals. With the availability of the empty scene measurements, background subtraction generates an image where the target can be easily identified, as shown in Fig. 1(b) and Fig. 2(b) for the far-target and close-target scenes, respectively.

Fig. 3 shows the results after scene reconstruction using (15) using the full datasets without background subtraction and with no preprocessing. To solve the convex optimization in
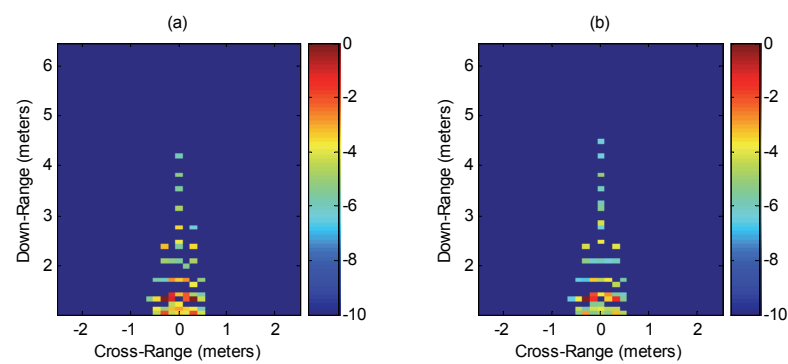

Fig. 3. TWRI image using CS with full data set and no preprocessing: (a) far target (b) close target.

(15), we use the Orthogonal Matching Pursuit (OMP) with the number of iterations set to 50 . It is evident from Fig. 3 that the wall mitigation techniques must be applied, as a preprocessing step, prior to CS.

For the CS approach in conjunction with wall mitigation, instead of using all space-frequency data, we use 128 uniformly selected frequencies and 17 uniformly selected array locations, which represent $4.5 \%$ of the total data volume. Applying the spatial filter for wall mitigation to the reduced data set provides the backprojection images shown in Fig. 4(a) and Fig. 4(c), which are degraded compared to their CS counterparts, depicted in Fig. 4(b) and Fig. 4(d). The maximum number of OMP iterations to obtain the CS based reconstructions was set equal to 20. Subtraction of the average value of (4) is performed for each of the reduced number of frequencies and across the available thinned array. From Fig. 4(b) and Fig. 4(d), we observe that the CS approach localizes the target accurately and provides less-cluttered images.

The advantage of CS over backprojection is also evident in Fig. 5, wherein the SVD method for wall mitigation is applied to the same reduced data set. The SVD is now applied to a reduced dimension B matrix, $128 \times 17$ instead of $728 \times 67$, when using the full data volume. In this case, we used (10) with $K=1$, i.e., we only removed the one-dimensional wall subspace. (a)

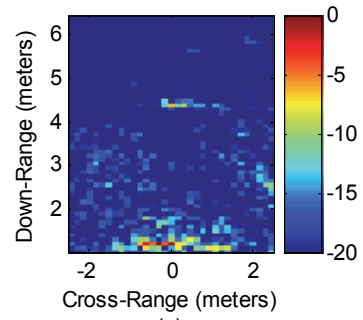

(c)

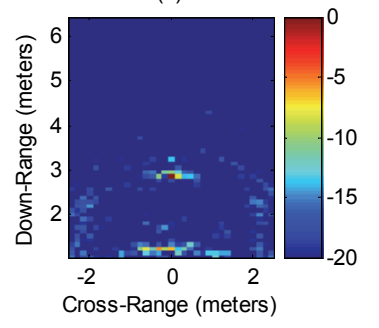

(b)

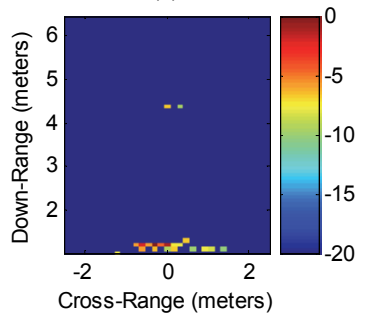

(d)

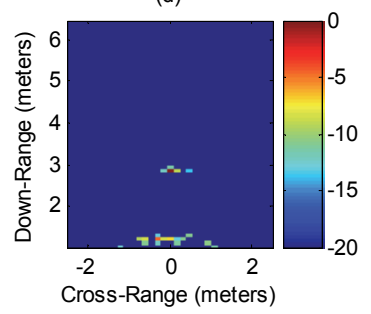

Fig. 4. Spatial Filtering with uniform reduced data: (a) Backprojection, far target; (b) CS, far target; (c) Backprojection, close target; (d) CS, close target. 
(a)
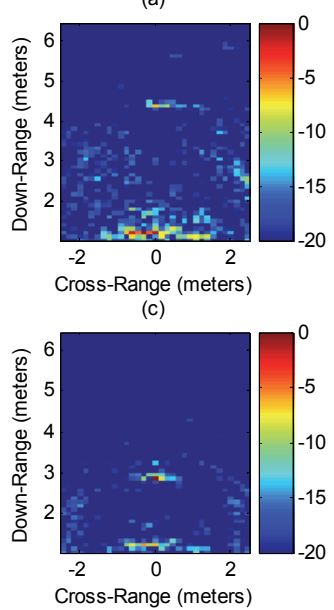

(b)

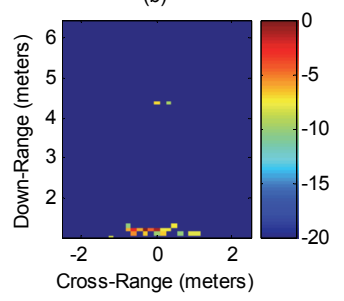

(d)

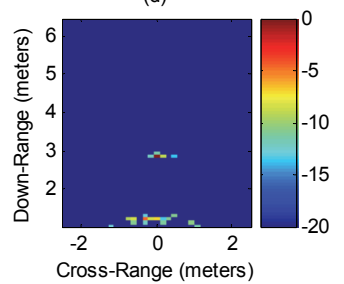

Fig. 5. SVD method with uniform reduced data: (a) Backprojection, far target;

(b) CS, far target; (c) Backprojection, close target; (d) CS, close target.

Alternatively, the number of measurements can be reduced through random selection of frequencies and antennas in lieu of uniform sample selections. Backprojection and CS methods are applied to the data which uses $4.5 \%$ randomly selected measurements. Fig. 6 and Fig. 7 show the backprojection and the CS images obtained with the spatial filter and the SVD methods, respectively. Each imaged pixel is the result of averaging 100 runs, with a different random frequency and aperture selection for each run. It is clear that the CS results provide much more focused and 'cleaner' images than those using backprojection.

\section{CONCLUSION}

In this paper, we examined the performance of the wall mitigation techniques for sparse scene reconstruction in through-the-wall radar imaging applications. We focused on the SVD based and the spatial filtering based wall mitigation methods. Using real data collected in a laboratory environment, we showed that the considered wall mitigation techniques maintain their proper performance when only a small subset of the full measurements is employed. Subsequent sparse reconstructions using the much reduced wall-clutter data successfully detected and accurately localized the targets.

\section{ACKNOWLEDGMENT}

This work was supported by ONR under grants N0001411-1-0576 and N0014-10-1-0455. E. Lagunas is partially supported by the fellowship FI-DGR-2011.

\section{REFERENCES}

[1] M. G. Amin, (Ed.), Special Issue on "Advances in indoor radar imaging”. J. Franklin Inst., vol 345, no. 6, pp. 556-722, Sep. 2008.

[2] M. G. Amin, Ed., Through-the-Wall Radar Imaging, CRC Press, Boca Raton, FL, 2010.

[3] M. Amin and K. Sarabandi, Eds., Special Issue on Remote Sensing of Building Interior, IEEE Trans. Geosci. Remote Sens., vol 47, no. 5, pp. 1270-1420, 2009

[4] A. Martone, K. Ranney, and R. Innocenti, "Automatic through the wall detection of moving targets using low-frequency UWB radar," in Proc. IEEE Int. Radar Conf., Washington D.C., May 2010, pp. 39-43. (a)
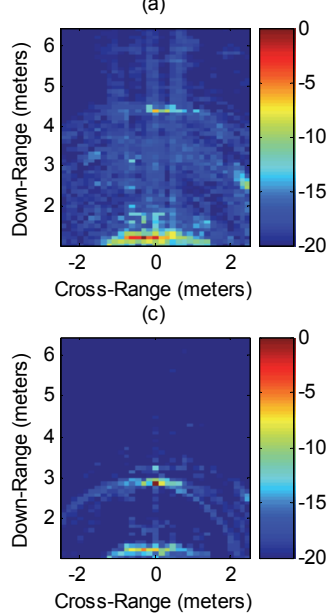

(b)

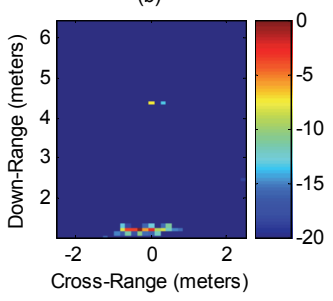

(d)

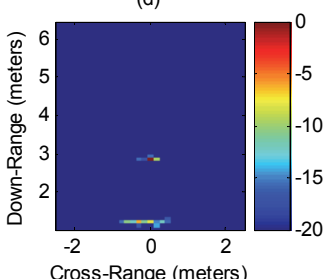

Fig. 6. Spatial Filter with random reduced data (averaged over 100 runs): (a) Backprojection, far target; (b) CS, far target; (c) Backprojection, close target; (d) $\mathrm{CS}$, close target (a)

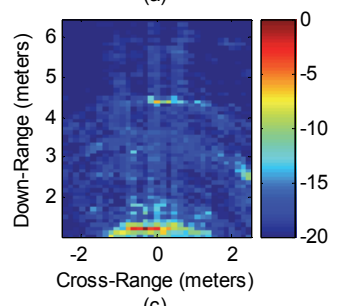

(c)

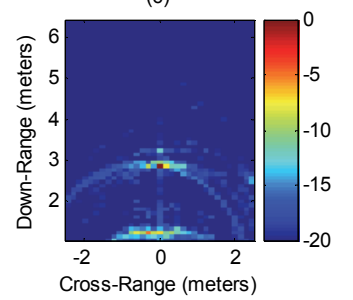

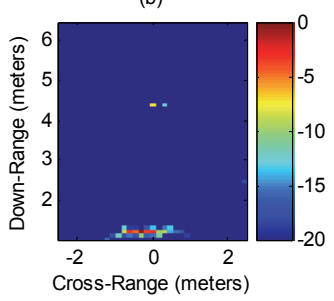

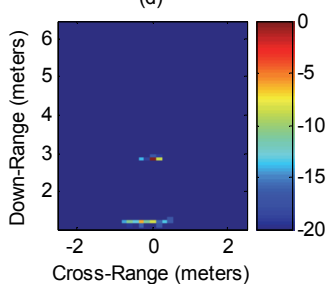

Fig. 7. SVD method with random reduced data (averaged over 100 runs): (a) Backprojection, far target; (b) CS, far target; (c) Backprojection, close target; (d) CS, close target.

[5] Y.-S. Yoon and M. G. Amin, "Spatial filtering for wall-clutter mitigation in through-the-wall radar imaging," IEEE Trans. Geosci. Remote Sens., vol. 47, no. 9, pp. 3192 - 3208, 2009.

[6] R. Chandra, A. N. Gaikwad, D. Singh, and M. J. Nigam, "An approach to remove the clutter and detect the target for ultra-wideband throughwall imaging," J. Geophys. Eng., vol. 5, no. 4, pp. 412-419, 2008.

[7] F. H. C. Tivive, A. Bouzerdoum, and M. G. Amin, "An SVD-based approach for mitigating wall reflections in through-the-wall radar imaging," in Proc. IEEE Radar Conf., Kansas City, MO, May 23-27, 2011, pp. 519-524.

[8] M. Dehmollaian and K. Sarabandi, "Refocusing through building walls using synthetic aperture radar," IEEE Transactions on Geoscience and Remote Sensing, vol. 46, no. 6, pp. 1589-1599, 2008.

[9] Y. Yoon and M. G. Amin, "Compressed sensing technique for highresolution radar imaging," in Proc. SPIE, vol. 6968,2008, pp. 69681A69681A-I0

[10] Q. Huang, L. Qu, B. Wu, and G. Fang, "UWB through-wall imaging based on compressive sensing," IEEE Trans. Geosci. Remote Sens., vol. 48, no. 3, pp. 1408-1415, 2010.

[11] M. Leigsnering, C. Debes, A.M. Zoubir, "Compressive sensing in through-the-wall radar imaging," in Proc. IEEE Int. Conf. Acoustics, Speech, Signal Process., Prague, Czech Republic, 2011, pp. 4008-4011.

[12] M. G. Amin and F. Ahmad, "Wideband synthetic aperture beamforming for through-the-wall imaging," IEEE Signal Process. Mag., vol. 25, no. 4, pp. 110-113, Jul. 2008. 